\title{
Topological analysis of the brain functional networks
}

\author{
Yoshinobu Shijo $^{\dagger}$, Shin'ichi Arakawa ${ }^{\dagger, \dagger \dagger}$, and Masayuki Murata ${ }^{\dagger,} \dagger^{\dagger \dagger}$ \\ $\dagger$ Graduate school of Information Science and Technology, Osaka University \\ 1-5, Yamadaoka, Suita, Osaka, 565-0871 Japan \\ $\dagger \dagger$ Center for Information and Neural Networks (CiNet), \\ National Institute of Information and Communications Technology(NICT), and Osaka University \\ 1-4, Yamadaoka, Suita, Osaka, 565-0871 Japan \\ $\dagger\{$ y-shijo, arakawa, murata\}@ist.osaka-u.ac.jp
}

\begin{abstract}
The Internet is one of the largest artificial network in the world, and it is desired to make the large-scale complex Internet into higher quality. Clues to the high quality can be found in human brain functional networks (BFNs) that show various functions at high level. In this paper, we analyze the topological structure and reveal advantages of BFNs from communication network perspective. Results of analysis show that BFNs have properties of fractality and disassortativity, and many superior paths at module-level topology.
\end{abstract}

\section{Categories and Subject Descriptors}

C2.1 [Computer-Communication Networks]: Network Architecture and Design—network communications, network topology

\section{General Terms}

Measurement

\section{Keywords}

Brain Functional Networks, Fractal, Internet, Topological Structure, Communication Quality

\section{INTRODUCTION}

The Internet is one of the largest artificial network in the world consisted of interconnections of many Autonomous Systems (ASes). It plays an important role as social infrastructures such as shopping, banking and trading systems. It is desired to make the large-scale complex Internet into higher quality because it becomes more important year after year. The high quality here means necessary properties for comfortably using and persistently managing the Internet,

Permission to make digital or hard copies of all or part of this work for personal or classroom use is granted without fee provided that copies are not made or distributed for profit or commercial advantage and that copies bear this notice and the full citation on the first page. To copy otherwise, to republish, to post on servers or to redistribute to lists, requires prior specific permission and/or a fee.

BIICT 2014, December 01-03, Boston, United States

Copyright @ 2015 ICST 978-1-63190-053-2

DOI 10.4108/icst.bict.2014.257881 such as high communication performance, high energy efficiency, and robustness against node and link failures and traffic changes. ASes, however, construct connections to other ASes selfishly, not considering the global structure of the Internet. It has been pointed out that a lot of traffic will concentrate on some ASes such as Hyper Giant in the future [13]. That is why the Internet is now on the way to fragileness.

Our research group focuses on human brain functional networks (BFNs) as clues to make the large-scale complex Internet into higher quality. BFNs show a wide variety of functions and exhibit human's rich behaviors. These functions are not innate, but acquired on the process of human's autonomous growth from child to adult. BFNs can manage and control itself with very small energy consumption by optimizing itself depending on the stage of growth [6]. In addition, it has been revealed that BFNs are organized for ensuring high efficiency of global communication within the overall network [16]. The Internet also grows and evolves autonomously as well as BFNs. However, total energy consumption in the network increases exponentially with enlarging its scale [4]. Moreover, it is difficult to say communication in the Internet is highly efficient because, for example, packets loss occurs due to traffic concentration.

As researches for BFNs' properties, many researchers generate brain functional network topology by using brain measurement data obtained from functional Magnetic Resonance Imaging (fMRI), and analyze the voxel-level topology with graph theory $[5,7,9,12]$. These results show BFNs have four fundamental properties: (1) power-law degree distribution; (2) small-world property where average hop count between nodes is small against the network scale; (3) hierarchical modular structure where nodes are tightly connected locally, and modules are connected in a hierarchical manner; and (4) fractality. Fractality is thought to be essential to attain coexistence of highly modular structure with broad global integration. That is, functional modules are sufficiently isolated to achieve independent computations and globally connected to achieve integration of each function.

Fractality is a self-similar property or repeating patterns at every scale. Originally, fractal is a geometric concept, but is extended for network topology [14]. A network topology is fractal when fractal dimension $D_{B}$ in the topology is finite value. For calculating $D_{B}$, box-counting algorithm is widely used. This algorithm tiles whole nodes in the network topology by "boxes" with size $l_{B}$. The size of box is defined by 
the maximum hop count between all node pairs in the box is less than $l_{B}$. Then, the fractal dimension $D_{B}$ is calculated by following relationship,

$$
N_{B}\left(l_{B}\right) / N \sim l_{B}^{D_{B}},
$$

where $N_{B}\left(l_{B}\right)$ is the minimum number of boxes and $N$ is the number of nodes in the network topology. When the value of $D_{B}$ is finite, the relation between box size $l_{B}$ and number of boxes $N_{B}\left(l_{B}\right)$ is scale-invariant. In other words, fractality in network topology is the property of preserving the proportion of the number of boxes to the box size. AS-level topology of the Internet shares two properties with BFNs in terms of having power-law degree distribution and small-world $[8,10]$. On the other hand, it does not have hierarchical modular structure and fractality [15]. We therefore analyze the BFNs' topological structure and its advantages.

The rest of this paper is organized as follows. Section 2 shows results of analysis of the topological structure of brain functional networks, and Section 3 shows its path quality. In Section 4, we conclude this paper and refer to future works.

\section{ANALYSIS OF THE TOPOLOGICAL STRUCTURE OF BRAIN FUNCTIONAL NETWORKS}

For the purpose of making the Internet higher quality by incorporating the BFNs' properties, we analyze quality of BFNs and clarify its advantages. Moreover, for the purpose of analyzing quality, we analyze the topological structure of brain functional networks. In this section, therefore, we reveal BFNs' topological structure, and give schematic view of the brain functional network topology.

\subsection{Topology Data}

By using fMRI, we can obtain time-series data of brain activities at each voxel. Voxel is a unit for fMRI measurement. Voxels are considered that they brain-functionally interacted with each other when they showed high-correlated brain activity transition. This is because brain functions are emerged as a result of brain activity aggregation. Thus, by regarding a voxel as a node and by constructing a link between voxels where correlation value for brain activity transition is more than given threshold, we can obtain the brain functional network topology that reflects brain-functionally interactions [5]. Note that the obtained topology may be disconnected depending on threshold. In this case, only most giant connected component is generally used for analysis.

We obtained the brain functional network topology for analysis by using method explained above. We measured subject's brain activities with fMRI. There is one male subject under resting-state conditions. The number of voxels was 80130. Taking into account topology scale, we set the threshold to 0.95 , and obtained the topology with 11420 nodes and 44049 links. Here after, we call this topology as voxel-level topology.

\subsection{Decomposition of Voxel-level Topology}

It is difficult to analyze the topological structure of the voxel-level topology because its topology scale is very large. To resolve this problem, we focus on hierarchical modular structure brought by fractality of BFNs. Module-level and inner-module topology at various grain scale can be obtained by regarding module as a node, and we mainly analyze these
Table 1: \# of nodes and links at each Path

\begin{tabular}{|c|c|c|}
\hline Path & \# of nodes & \# of links \\
\hline 0 & 11420 & 44049 \\
1 & 1989 & 3007 \\
2 & 432 & 654 \\
3 & 179 & 288 \\
4 & 146 & 236 \\
\hline
\end{tabular}

topologies. Analyzing module-level topology enable us to focus deeply on "functions" which are the essentials of BFNs. A voxel is merely a measuring point of fMRI and it does not directly relate to brain functions. The module, on the other hand, is aggregation of nodes, so this is highly correlated to brain functions.

For obtaining module-level topology at various hierarchies, we first detect hierarchical modular structure. We use socalled "Louvain method [3]" for this purpose. This method can find module identifier $M_{i}(h)$ of node $i$ at Path (hierarchy) $h^{1}$. We obtained module-level topology at Path $h$ by following procedures:

Step.1 Generate initial topology. The number of nodes is set to the number of modules at Path $h$. The number of links is set to zero.

Step.2 Construct a link between nodes $M_{i}(h)$ and $M_{j}(h)$ when a link exists between node $i$ and node $j$ at voxellevel topology and satisfies $M_{i}(h) \neq M_{j}(h)$.

Note that we call voxel-level topology as module-level topology at Path 0 for convenience.

Inner-module topology at Path $h$ consists of voxel-level nodes with same module identifier at Path $h$. That is, innermodule topology can be obtained as subgraph of the voxellevel topology. For each Path-level, the voxel-level topology can be decomposed into a set of inner-module topologies. We distinguish inner-module topologies by the rank of module size in descending order of the size.

As an example, Figure 1 is the illustration of decomposition of voxel-level topology with 26 nodes. A group of nodes surrounded by a circle is a module. The nodes in the same module have same module identifier. This voxel-level topology contains 6 modules at Path1, 3 modules at Path2, and 1 module at Path3. Module-level topology at Path1 and Path2 obtained by applying procedures explained above is illustrated at the left bottom of the Figure 1. Even if there are multiples links between modules at voxel-level topology, they are regarded as single link at module-level topology. Thus we can obtain module-level topology with 6 nodes and 7 links at Path1 and with 3 nodes and 2 links at Path2. On the other hand, inner-module topologies at Path2 is illustrated at the right bottom of the Figure 1 . We can obtain 3 inner-module topologies at Path2 because there are 3 modules at Path2. The number of nodes of each inner-module topology is 11,9 , and 6 from the left. Therefore, the rank of module size is assigned 1,2 , and 3 from the left.

Table 1 shows the number of nodes and links of the obtained module-level topology. Figure 2 show $l_{B}$ versus $N_{B}$ distribution with box-counting analysis, and Figure 3 shows degree distribution of module-level topology. Figures reveal that the module-level topology has fractality, and its degree

${ }^{1}$ We call the level of hierarchy as "Path" by following [3]. 


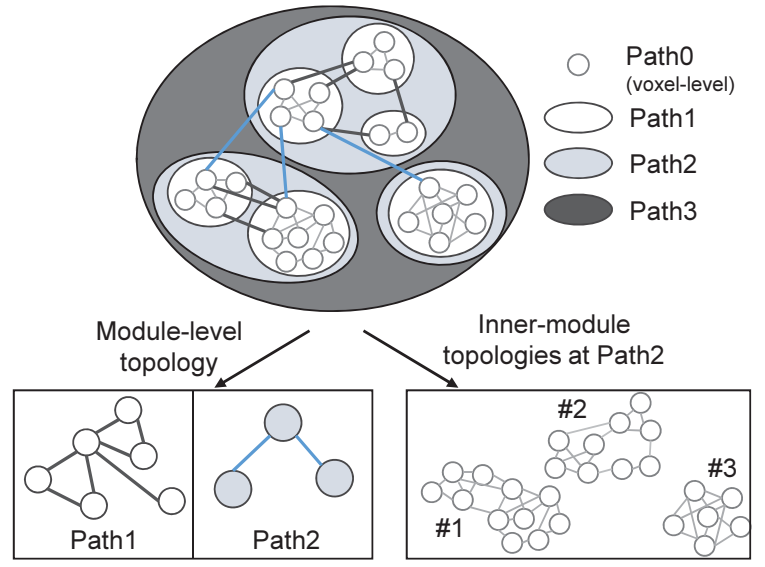

Figure 1: Illustration of decomposition of voxel-level topology

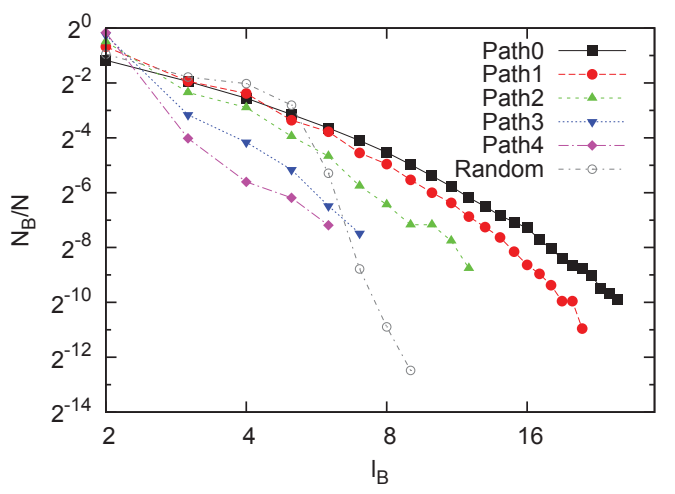

Figure 2: $l_{B}$ versus $N_{B}$ distribution of module-level topology with box-counting analysis

distribution follows power-law. In addition, we examined degree distribution of the inner-module topology, and confirmed that the inner-module topology with sufficient number of nodes also has power-law degree distribution.

\subsection{Metrics}

We decomposed the large-scale voxel-level topology into the module-level and inner-module topology in the previous subsection. By using it, complex topological structure is also decomposed into module-level, inner-module and intermodule structure. Here we use following three metrics to reveal these three topological structure.

\section{Degree-Correlation.}

This is correlation of degree of node which constructs link. We use two methods in [11] and [15]. Method in [15] can analyze in detail, on the other hand it is difficult to apply this method to small-scale topology. So we do not apply this method to inner-module topologies since many of them are small-scale, and only apply to module-level topologies. Method in [11] is applied to both of module-level and innermodule topology. We explain overview of these methods below.

Method in [15] can reveal degree of node pairs which have

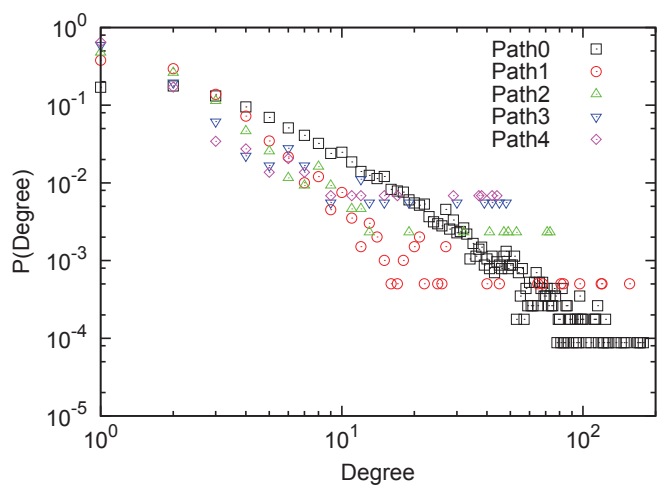

Figure 3: Degree distribution of module-level topology

more (or fewer) links compared to corresponding random topology. Degree-correlation $R\left(k_{1}, k_{2}\right)$ is defined as:

$$
R\left(k_{1}, k_{2}\right)=P\left(k_{1}, k_{2}\right) / P_{r}\left(k_{1}, k_{2}\right),
$$

where the value $P\left(k_{1}, k_{2}\right)$ is probability of finding a node with $k_{1}$ links connected to a node with $k_{2}$ links. The value $P_{r}\left(k_{1}, k_{2}\right)$ is random uncorrelated counterpart, which is obtained by random swapping of the links preserving the degree distribution.

Method in [11] can reveal how many links there are between high or low degree nodes. Degree-correlation $l$ is defined as:

$$
l=\left(L-L_{\min }\right) /\left(L_{\max }-L_{\min }\right),
$$

where $L=\sum_{(i, j) \in E} k_{i} k_{j} . L_{\max }$ and $L_{\min }$ are maximum and minimum value of $L$ among all topology with same degree sequence. The value of $l$ is ranged from 0 to 1 . When value of $l$ of the topology takes high, high-degree nodes tend to be connected with other high-degree nodes and low-degree nodes tend to be connected with other low-degree nodes. When value of $l$ of the topology takes low, high-degree nodes tend to be connected with low-degree node.

\section{Correlation between module size and the number of inter-module links.}

We calculate the number of links between modules. To reveal which module has many or few links to other modules, we introduce the module size. The size of module is defined as big if corresponding inner-module topology has a lot of nodes.

\section{Correlation between node degree and the number of inter-module links.}

To reveal which degree of node at voxel-level has many or few inter-module links, we calculate the number of intermodule links among all node pairs based on node degree. Note that node degree is defined by degree in the innermodule topology, that is, node degree is $k_{i n}$ if there is a node with $k_{\text {out }}$ links to other modules and $k_{i n}$ links to same module.

\subsection{Results of Analysis}

As results of analysis, we got similar results over all Paths. Here we refer to the only topology at Path2 on behalf of the 


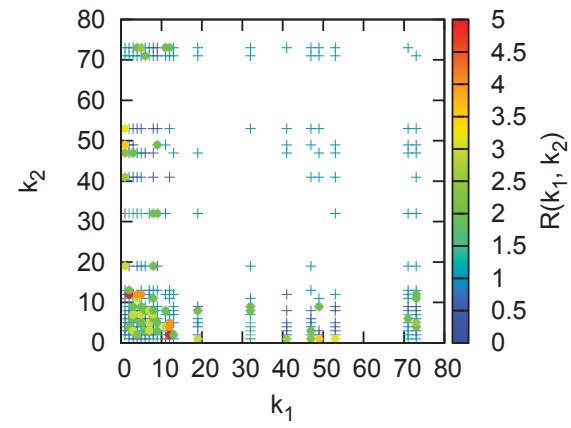

Figure 4: Degree-correlation of the module-level topology at Path2

topology at other Paths.

\subsubsection{Degree-Correlation}

Figure 4 shows degree-correlation of the module-level topology by the definition in [15]. Both axes are node-degree $k_{1}$ and $k_{2}$, and the color of plots shows the value of $R\left(k_{1}, k_{2}\right)$. The plots in the figure are bigger where the value of $R\left(k_{1}, k_{2}\right)$ is more than 2. Most of plots are located at the area where both $k_{1}$ and $k_{2}$ are small, or one is small and the other is big. It shows that the module-level topology have property of hub-hub repulsion or disassortativity.

Figure 5 shows degree-correlation of inner-module topologies. Here, we use the definition in [11] instead of [15] for calculating degree-correlation. This is because most of inner-module topologies are too small to apply the method in [15]. An x-axis is rank of module size and y-axis is degreecorrelation $l$. The figure shows the inner-module topology with large number of nodes has high degree-correlation. Average value of degree-correlation $l$ for inner-module topology with more than 100 nodes is 0.726 . Note that degreecorrelation $l$ of module-level topology is 0.561 . This means inner-module topology has more links between high-degree nodes than the module-level topology. Note that the value of degree-correlation $l$ varies where the rank of module size is more than 100 . When the number of nodes is relatively small, the difference between $L_{\max }$ and $L_{\min }$ in equation (3) becomes small. Therefore the value of $l$ greatly changes by the slight difference of node pairs which construct link. In this case, the value of $l$ cannot reflect accurate degreecorrelation, so modules where the rank of module size is more than 100 are not worth consideration.

\subsubsection{Correlation between module size and the num- ber of inter-module links}

Figure 6 shows correlation between module size and the number of inter-module links. Both axes are the rank of module size, and the color of plots reflects the number of inter-module links. Most of plots are located at the area where the value of one axis is low, which means modules with large number of nodes construct many links to various modules. On the other hand, a few plots are located at the area where the value of both axes is not low, which means modules with medium or small number of nodes construct a few links to small variety of modules. These results suggest most of modules have two types of inter-module links; one for connecting with large modules and the other for con-

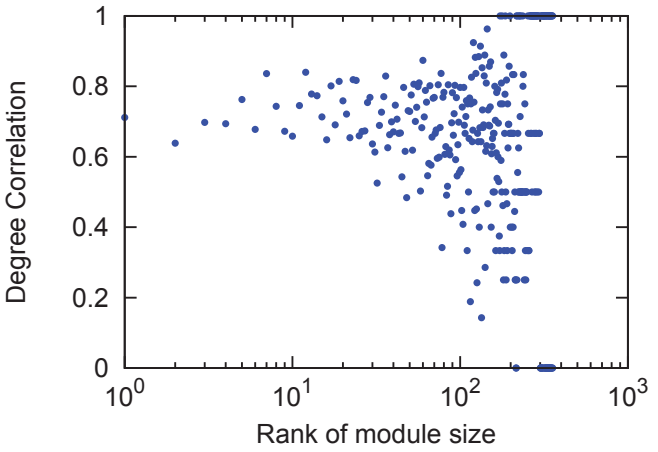

Figure 5: Degree-correlation of the inner-module topologies at Path2

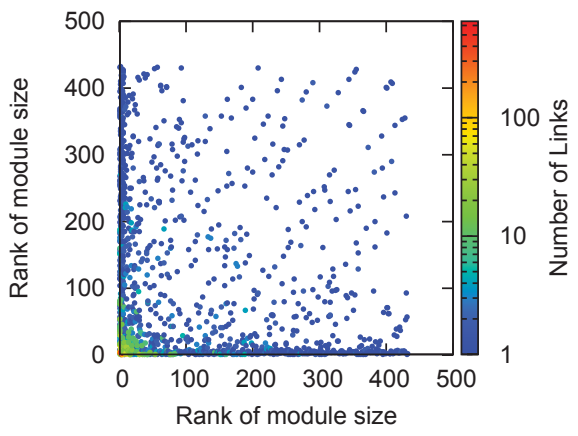

Figure 6: Correlation between module size and the number of inter-module links

necting with medium or small modules. The number of the former links is more than that of the latter links.

\subsubsection{Correlation between node degree and the num- ber of inter-module links}

Figure 7 shows correlation between node degree and the number of inter-module links. Both axes are the value given by dividing degree of node $i$ which have inter-module links by the average degree of inner-module topology which node $i$ belongs to. The color of plots reflects the number of intermodule links. The plots are concentrated in the area of around $(1,1)$. Therefore inter-module links exist between nodes whose degree is average degree in each inner-module topology.

\subsubsection{Schematic view of the brain functional network topology}

We show schematic view of the brain functional network topology in Figure 8 that reflects topological structure as summary of analysis in this section. The thickness of lines between modules indicates the number of inter-module links. The node filled in the black at voxel-level corresponds to hub node, or node with high degree, in the module.

According to analysis results of topological structure and schematic view, it is conceivable that BFNs can globally perform efficient communication via hub node at modulelevel topology. We consider BFNs are also able to locally perform such communication thanks to fractality at modulelevel topology. 


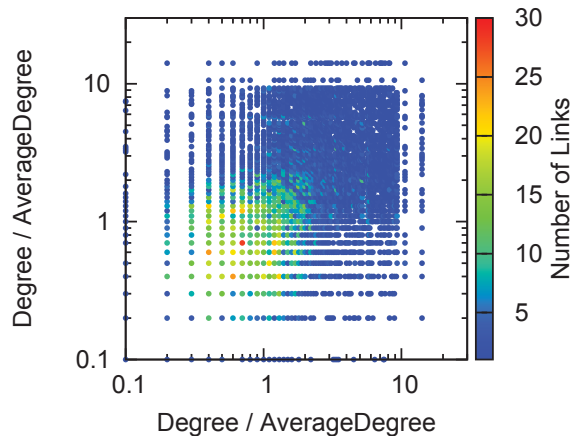

Figure 7: Correlation between node degree and the number of inter-module links

\section{PATH QUALITY EVALUATION}

For applying BFNs to information network, it is important to reveal impacts of the fractality on communication quality. In this section, we focus on path quality as communication quality at module-level topology, and evaluate it.

\subsection{Topologies for comparison}

In this paper, we evaluate path quality by comparing hop count of various topologies. We examined following topologies obtained by network generation models for comparison. These models are used in the field of neuroscience and/or reflect a part of structural properties of BFNs.

- Random model

- Barabási-Albert (BA) model [2] which reflects the structural property of power-law degree distribution.

- Watts-Strogatz (WS) model [17] which reflects the integration of local optimality and global efficiency [16].

- Waxman model [18] which reflects the property that functional modules in anatomically close position are densely connected [1].

We evaluate the module-level topology from Path1 to Path4 respectively. The topologies for comparison are generated with models so that the number of nodes and links of those corresponds to module-level topology at each Paths. Note that WS and Waxman model have several parameters. WS model have parameter $p$ which is proportion of random swapping of links. We calculated the average hop count $H(p)$ and clustering coefficient $C(p)$ against $p$, and obtained the result shown in Figure 9. Regarding high clustering coefficient as local optimality and the low average hop count as global efficiency, the value of $p$ should be around 0.1 to integrate high clustering coefficient and the low average hop count. Therefore we assign parameter $p$ to 0.1 for WS model. On the other hand, Waxman model have parameters $\alpha$ and $\beta$. They influence the probability to have a link between node $u$ and node $v$ defined by

$$
P(u, v)=\alpha e^{-d(u, v) / \beta L},
$$

where $d(u, v)$ is the distance between $u$ and $v$, and $L$ is the maximum distance between any two node. The value of $\alpha$ affect the number of links of the topology. When the value of $\alpha$ is higher, the generated topology has more links.

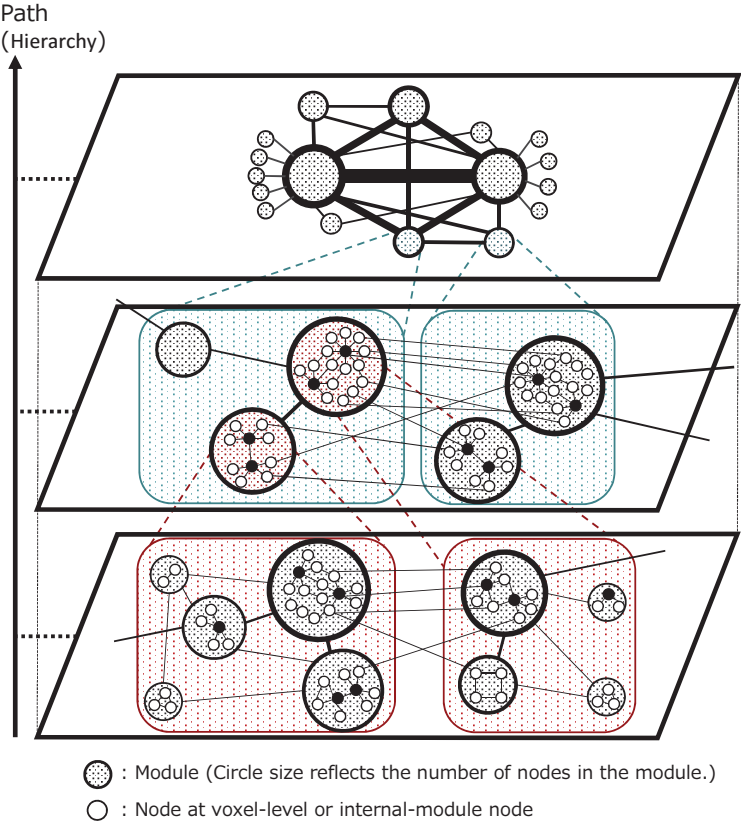

Figure 8: Schematic view of the brain functional network topology that reflects analysis results of topological structure

However, the number of links of the comparison topology corresponds to that of module-level topology in this paper. Hence, we assign $\alpha$ to 1.0 and modify WS model to generate topology with given number of links. The value of $\beta$ affect the probability to have a link between nodes with certain distance. When the value of $\beta$ is lower, the less links are constructed between nodes with long distance, or nodes with short distance have more links. Therefore, we assign $\beta$ to 0.1 for reflecting property that nodes in close position are densely connected.

\subsection{Results}

We take a hop count as path quality. This is because the hop count can reflect the topological static performance. We also focus on the redundancy of the paths. For this purpose, we use Yen's $K$ shortest paths algorithm [19] for deriving paths, and calculate the hop count from shortest path to 300th path. Defining $H_{K}$ as the average hop count of $K$-th shortest path between arbitrary node pair, we take distribution of $H_{1}, H_{2}, \ldots$ and $H_{300}$ as metrics for path quality.

Table 2 show the values of $H_{1}, H_{300}$ and average and variance from $H_{1}$ to $H_{300}$ at each Path. From this table we observe that BFNs take the minimum average value in evaluated topologies at every Path. This means BFNs have high quality paths in terms of the hop count. In the same way, BFNs also take the minimum variance. This means BFNs have a lot of paths with similar quality. These results show BFNs have many superior paths compared to comparison topologies at module-level.

We also observe that BA-model network take the second minimum average value. BFNs and BA-model network differ from the other evaluated topologies in degree distribution. Figure 10 shows BFNs and BA-model network follow power- 


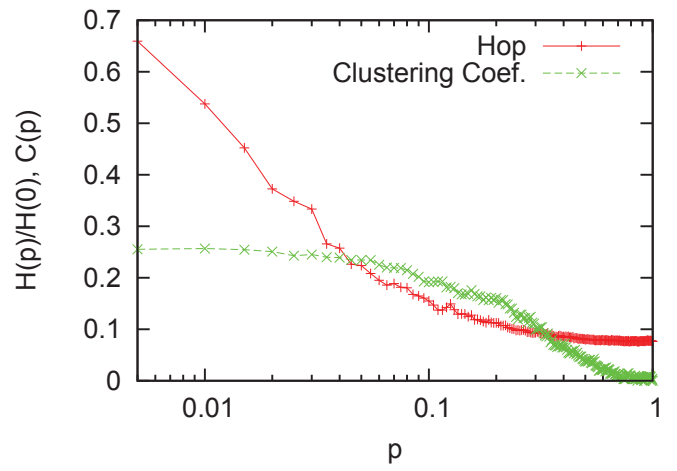

Figure 9: The value of the average hop count $H(p)$ and clustering coefficient $C(p)$ against $p$ in topology obtained by WS model

Table 2: Result of path quality analysis.

\begin{tabular}{|c|c|c|c|c|c|c|}
\hline & & Brain & BA & Rand. & WS & Waxman \\
\hline \multirow{5}{*}{ Path1 } & $H_{1}$ & 5.68 & 5.07 & 7.52 & 14.50 & 10.99 \\
& $H_{300}$ & 8.33 & 9.04 & 14.17 & 18.90 & 15.78 \\
& Avg. & 7.85 & 8.35 & 13.11 & 17.96 & 14.88 \\
& Var. & 0.20 & 0.42 & 1.11 & 0.70 & 0.69 \\
\hline \multirow{5}{*}{ Path2 } & $H_{1}$ & 3.79 & 4.02 & 5.90 & 12.03 & 6.01 \\
& $H_{300}$ & 7.14 & 8.81 & 12.53 & 17.70 & 12.52 \\
& Avg. & 6.48 & 7.90 & 11.43 & 16.63 & 11.42 \\
& Var. & 0.30 & 0.68 & 1.13 & 0.99 & 1.11 \\
\hline \multirow{5}{*}{ Path3 } & $H_{1}$ & 2.91 & 3.46 & 4.73 & 8.35 & 4.56 \\
& $H_{300}$ & 6.58 & 8.71 & 11.27 & 14.46 & 11.21 \\
& Avg. & 5.81 & 7.69 & 10.16 & 13.28 & 10.10 \\
& Var. & 0.40 & 0.82 & 1.10 & 1.21 & 1.13 \\
\hline \multirow{5}{*}{ Path4 } & $H_{1}$ & 2.71 & 3.32 & 4.45 & 8.66 & 4.41 \\
& $H_{300}$ & 6.49 & 8.71 & 10.89 & 14.46 & 10.63 \\
& Avg. & 5.70 & 7.64 & 9.78 & 13.29 & 9.55 \\
& Var. & 0.44 & 0.87 & 1.10 & 1.16 & 1.01 \\
\hline
\end{tabular}

law while the other topologies do not. The network with power-law degree distribution can communicate effectively via hub node. Table 2 reflects this fact because $H_{1}$ and the average value over all paths are small in BFNs and BAmodel network. On the other hand, the hop count variance of BFNs is half or less than that of BA-model network. This indicates BFNs have more paths whose hop count is close to average. Therefore we assume that fractality which is unique to BFNs contributes to high communication efficiency.

We have concluded path quality taking whole network into consideration before here. However, in the communication on the information network, it is necessary to focus on path quality of node pair with the long hop count. Assume that node pair with short hop count communicate by using detour paths due to, for example, link failure or traffic congestion along the shortest path. In this case, the path for this communication is replaced by paths with longer hop count distance. Here we extract node pair whose hop count equals to topology diameter $\times 0.8$ from each evaluated topologies. Then distribution of $H_{K}$ is calculated for extracted node pair. Figure 11 shows the hop count transition against $K$. An $\mathrm{x}$-axis is the value of $K$ and $\mathrm{y}$-axis is the hop count at $K$-th shortest path. The value in parentheses shows the

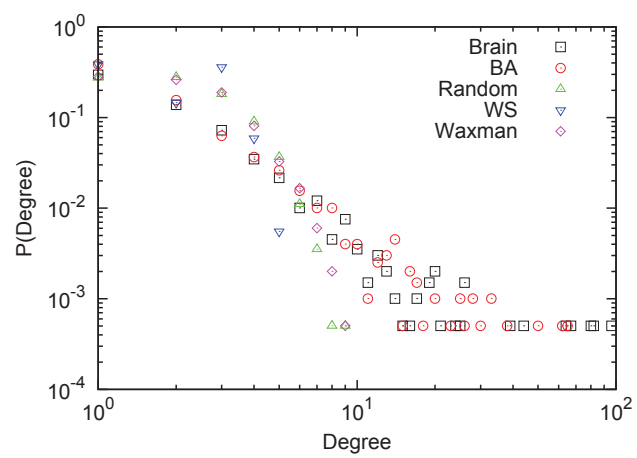

Figure 10: Degree distribution of evaluated topologies

hop count of extracted node pair. We omit results of network at Path3 and Path4 since they show similar result to Path1. From this figure, we observe that BFNs can keep down change of $H_{K}$ most. This means BFNs have the best quality when considering only the paths with the long hop count. On the other hand, BA-model network, which has high quality paths in whole network, is inferior to Waxmanmodel network at Path1. Therefore we consider that fractality has good impact on communication on information network.

\section{CONCLUSION AND FUTURE WORKS}

In this paper, we focus on BFNs as clues for making the Internet into higher quality. We first analyze topological structure. The results of analysis show BFNs have fractality and power-law degree distribution at module-level topology. The results also shows module-level topology is disassortative, on the other hand inner-module topology is more assortative. The links at module-level mainly exist between large modules. The inter-module links at voxel-level exist between nodes whose degree is average degree in each inner-module topology. Second we reveal the path quality of BFNs. The results suggest that fractality of BFNs contributes to high communication efficiency not only in whole network but also in environment of information network. Therefore we obtain a perspective of making the Internet higher quality by incorporating BFNs' fractality.

As future works, we will reveal additional advantages of BFNs such as robustness against node or module failures. Furthermore, although we use model-based topology as comparison topologies in this study, we will use other topologies such as random-rewired brain functional network topology. This approach enable us to reveal relationship between topology property and communication quality in further detail.

\section{ACKNOWLEDGEMENT}

We would like to thank Dr. Tetsuya Shimokawa (NICT) who offered valuable discussion and support for obtaining data of brain functional networks.

\section{REFERENCES}

[1] A. F. Alexander-Bloch, P. E. Vértes, R. Stidd, F. Lalonde, L. Clasen, J. Rapoport, J. Giedd, E. T. 

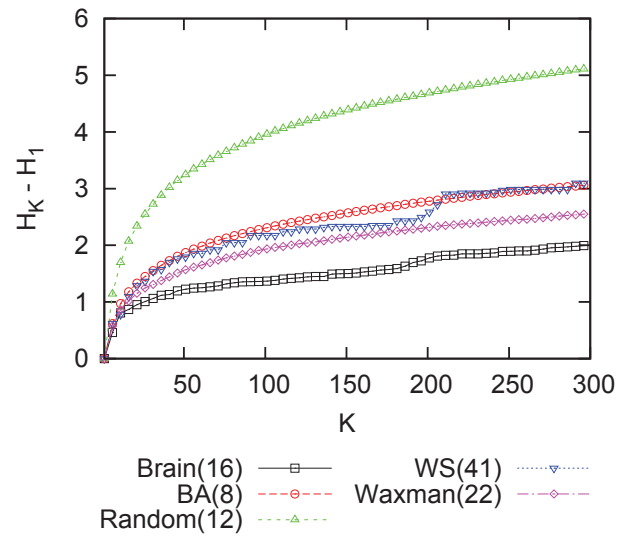

(a) Path1

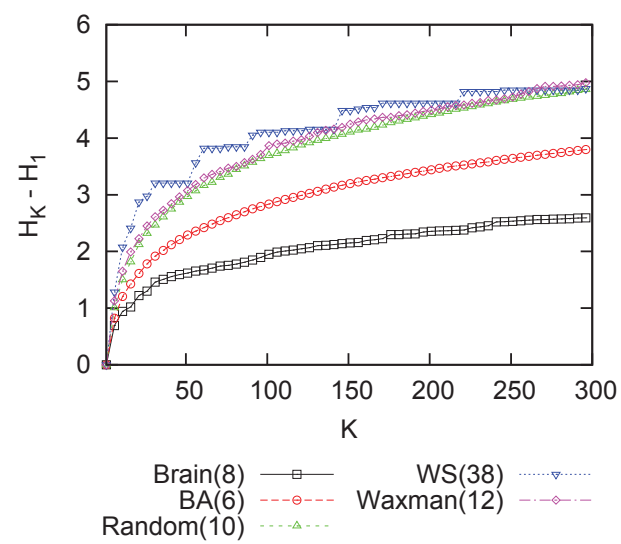

(b) Path2

Figure 11: Result of path quality analysis considering hop count distance between nodes

Bullmore, and N. Gogtay. The Anatomical Distance of Functional Connections Predicts Brain Network Topology in Health and Schizophrenia. Cerebral Cortex, 23(1):127-138, Jan. 2012.

[2] A.-L. Barabási and R. Albert. Emergence of Scaling in Random Networks. Science, 286(5439):509-512, Oct. 1999.

[3] V. Blondel, J. Guillaume, R. Lambiotte, and E. Lefebvre. Fast unfolding of communities in large networks. Journal of Statistical Mechanics, pages 1-12, July 2008.

[4] R. Bolla, R. Bruschi, F. Davoli, and F. Cucchietti. Energy efficiency in the future internet: A survey of existing approaches and trends in energy-aware fixed network infrastructures. Communications Surveys 85 Tutorials, IEEE, 13(2):223-244, May 2011.

[5] E. Bullmore and O. Sporns. Complex brain networks: graph theoretical analysis of structural and functional systems. Nature Reviews Neuroscience, 10(3):186-198, Mar. 2009.

[6] E. Bullmore and O. Sporns. The economy of brain network organization. Nature Reviews Neuroscience, 13(5):336-349, Apr. 2012.
[7] V. M. Eguiluz, D. R. Chialvo, G. A. Cecchi, M. Baliki, and V. V. Apkarian. Scale-free brain functional networks. Physical Review Letters, 94(1):1-4, Jan. 2005.

[8] M. Faloutsos, P. Faloutsos, and C. Faloutsos. On power-law relationships of the internet topology. ACM SIGCOMM Computer Communication Review, 29(4):251-262, Aug. 1999.

[9] L. K. Gallos, H. A. Makse, and M. Sigman. A small world of weak ties provides optimal global integration of self-similar modules in functional brain networks. Proceedings of the National Academy of Sciences, 109(8):2825-2830, Feb. 2012.

[10] S. Jin and A. Bestavros. Small-world characteristics of internet topologies and implications on multicast scaling. Computer Networks, 50(5):648-666, Apr. 2006.

[11] L. Li, D. Alderson, W. Willinger, and J. Doyle. A first-principles approach to understanding the internet's router-level topology. ACM SIGCOMM Computer Communication Review, 34(4):3-14, Aug. 2004.

[12] D. Meunier, R. Lambiotte, A. Fornito, K. Ersche, and E. T. Bullmore. Hierarchical modularity in human brain functional networks. Frontiers in Neuroinformatics, 3(37):1-12, Oct. 2009.

[13] M. Palacin, M. Oliver, J. Infante, S. Oechsner, and A. Bikfalvi. The impact of content delivery networks on the internet ecosystem. Journal of Information Policy, 3:304-330, July 2013.

[14] C. Song, S. Havlin, and H. Makse. Self-similarity of complex networks. Nature, 433:392-395, Jan. 2005.

[15] C. Song, S. Havlin, and H. Makse. Origins of fractality in the growth of complex networks. Nature Physics, 2:275-281, Apr. 2006.

[16] M. P. van den Heuvel, C. J. Stam, R. S. Kahn, and H. E. Hulshoff Pol. Efficiency of functional brain networks and intellectual performance. The Journal of Neuroscience, 29(23):7619-7624, June 2009.

[17] D. Watts and S. Strogatz. Collective dynamics of 'small-world' networks. Nature, 393(6684):440-442, June 1998.

[18] B. M. Waxman. Routing of multipoint connections. IEEE Journal on Selected Areas in Communications, 6(9):1617-1622, Dec. 1988.

[19] J. Y. Yen. Finding the K Shortest Loopless Paths in a Network. Management Science, 17(11):712-716, July 1971. 